\title{
CAPÍTULO 34: AVALIAÇÃO DA QUALIDADE DE LEITE COMERCIALIZADO INFORMALMENTE NO BREJO PARAIBANO
}

\section{CHAPTER 34: EVALUATION OF THE QUALITY OF MILK COMMERCIALIZED INFORMALMENTE IN THE PARAIBANO BREJO}

\author{
Laíza Soliely Costa Gonçalves ${ }^{1}$; Kimberly Cristina da Costa Pereira²; Aliou Toro Lafia ${ }^{3}$ Luiza Soliana \\ Costa Gonçalves ${ }^{4}$; Fabiana Augusta Santiago Beltrão ${ }^{5}$
}

\begin{abstract}
Resumo
A comercialização informal de leite in natura ainda é uma prática comum, apesar de ser proibida por lei, ainda é vista nas cidades do interior. O objetivo deste trabalho foi avaliar a qualidade microbiológica e físico-química de leite cru comercializado informalmente nas cidades de Solânea PB e Bananeiras PB no Brejo Paraibano. Foram realizadas análises microbiológicas e físico-químicas de amostras coletadas em dez comércios dos municípios de Solânea e Bananeiras. conforme legislação. O experimento foi conduzido no Laboratório de Pesquisa e Desenvolvimento de Produtos Laticínios (PDLAT), no Laboratório de Microbiologia de Alimentos (LMA) e no Laboratório de Análise Físico-química de Alimentos (LAFQ), do CCHSA, Campus III - Bananeiras, da UFPB, durante 4 semanas. Resultando onde todas as amostras apresentaram-se fora dos padrões em relação à pelo menos um parâmetro de análises microbiológicas e físico-químicas, respectivamente. Concluiu-se que o leite comercializado cru nas cidades de Solânea e Bananeiras não atende aos requisitos microbiológicos e físico-químicos preconizados pela legislação vigente, apresentando diferenças que confirmam que o leite comercializado pode ser prejudicial à saúde e pode conter adulteração.
\end{abstract}

Palavras-Chave: Controle de qualidade; leite bovino; condições higiênico-sanitárias; qualidade do leite.

\begin{abstract}
The informal marketing of fresh milk is still a common practice, despite being prohibited by law, it is still seen in inland cities. The objective of this work was to evaluate the microbiological and physicalchemical quality of raw milk sold informally in the cities of Solânea PB and Bananeiras PB in Brejo Paraibano. Microbiological and physical-chemical analyzes were carried out on samples collected in ten stores in the municipalities of Solânea and Bananeiras. according to legislation. The experiment was conducted at the Laboratory of Research and Development of Dairy Products (PDLAT), at the Laboratory of Food Microbiology (LMA) and at the Laboratory of Physical-Chemical Analysis of Food (LAFQ), at CCHSA, Campus III - Bananeiras, at UFPB for 4 weeks. Resulting where all samples were found out of the standards in relation to at least one parameter of microbiological and physical-chemical analyzes, respectively. It was concluded that raw milk sold in the cities of Solânea and Bananeiras does not meet the microbiological and physico-chemical requirements recommended by current legislation, with differences that confirm that commercialized milk can be harmful to health and may contain adulteration.
\end{abstract}

Keywords: Quality control; bovine milk; hygienic-sanitary conditions; milk quality.

\footnotetext{
${ }^{1}$ Técnico em Agroindústria, Colégio Agrícola Vidal de Negreiros, Bacharel em Agroindústria Universidade Federal da Paraíba, laizasolielyc@gmail.com

2 Bel. em Agroindústria, Universidade Federal da Paraíba, kimberly_crys@hotmail.com

${ }^{3}$ Mestrando em Tecnologia de Alimentos, PPGTA, Universidade Federal da Paraíba, zime1990@gmail.com

${ }^{4}$ Bel. Química Industrial, Universidade Estadual da Paraíba, solianaa luiza@gmail.com

${ }^{5}$ Docente, Universidade Federal da Paraíba, fasb.15@hotmail.com
} 


\section{Introdução}

O leite bovino é um produto agropecuário de grande importância econômica para geração de emprego e renda no mundo, desempenhando o papel de alimento humano (FERREIRA et al., 2017). Segundo a Instrução Normativa $n^{\circ}$ 62, de 29 de dezembro de 2011, do Ministério da Agricultura, Pecuária e Abastecimento (MAPA), o leite é definido como o produto obtido da ordenha completa, ininterrupta, em condições de higiene, de vacas sadias, bem alimentadas e descansadas (BRASIL, 2011). Sendo denominado como leite cru refrigerado, o leite produzido em propriedades rurais, refrigerado e destinado aos estabelecimentos de leite e derivados sob serviço de inspeção oficial (BRASIL. 2018). O leite cru e in natura, é o leite que não sofreu o processamento térmico adequado, ou seja, é susceptível a presença de patógenos, não existindo a garantia de qualidade e segurança para o consumo (VIDAL; NETTO, 2018).

O leite é um alimento completo rico em macronutrientes e micronutrientes, tornando um alimento nutritivo e fundamental para a alimentação de indivíduos de todas as faixas de idades, desde da inclusão em dietas para crianças como para idosos. Devido a sua composição rica em nutrientes e compostos, é um produto propicio para uma grande diversidade, crescimento e desenvolvimento de micro-organismos tanto benéficos como patogénicos. O que por sua vez pode acarretar na probabilidade de desenvolver algum tipo de contaminação, e por esse motivo a higiene e obtenção de leite com qualidade é indispensável, assegurando a sua inocuidade junto ao consumidor. A qualidade do leite está relacionada a preocupação de técnicos e autoridades ligadas à área de saúde, principalmente, pelo risco de propagação de micro-organismos patogênicos relacionados com doenças de origem alimentar (VIEIRA et al, 2001; TIMM et al., 2003; TRONCO, 2003).

Uma forma de garantir o controle de qualidade do leite é através da utilização dos processos térmicos, como a refrigeração, pasteurização e a Ultra Pasteurização (UHT), a fim de inibir o crescimento desordenado de bactérias. Apesar que em algumas circunstâncias, enzimas possuem maior resistência a esses tipos de processos térmicos, prejudicando a qualidade do leite e seus derivados, como por exemplo, as bactérias psicrotróficas e sua atividade proteolítica (TRONCO, 2003; ORDÓÑES, 2005)

O consumo do leite in natura, ou seja, sem a aplicação de qualquer processo térmico, é habitual no interior da Paraíba assim como no Nordeste, devido a hábitos culturais, regionais e sociais, utilizando da justificativa de que o leite obtido diretamente do produtor é mais saudável que o industrializado, além da forma de aquisição ser de baixo custo comparado a venda de leite formal, vale salientar que a comercialização informal de leite no Brasil é proibida desde 1952 (MOLINA et al, 2015; MOTTA et al, 2015)

Visto que a fiscalização para esse produto de forma informal é incipiente, ou seja, não há garantia de que os padrões microbiológicos e físico-químicos exigidos pela Instrução Normativa $\mathrm{n}^{\circ} 76$ e 77 de 26 de dezembro 2018, do Ministério da Agricultura, Pecuária e Abastecimento, sejam executados pelos produtores e comerciantes informais. A não inspeção do leite favorece a adulteração por parte dos 
produtores, os quais utilizam dessa prática repentinamente, por exemplo, através da fraudação com a retirada da gordura, a adição de água, a adição de neutralizantes e conservantes, práticas essas comuns para aumentar o rendimento do volume do leite e/ou mascarar problemas como deterioração causados pelas inadequadas ou inexistentes de boas práticas de fabricação e refrigeração, já que esse produto não é submetido e nem assegurado a qualquer exigência de controle de qualidade por legislação (MENDES et al., 2010; MONTANHINI; HEIN, 2013; TRINDADE et al, 2018; BRASIL, 2018).

O leite se obtido e manipulado em condições inadequadas e comercializado informalmente se torna uma preocupação para a saúde pública, pois gera um risco potencial para consumidor que consome na forma in natura ou na forma de seus derivados, podendo causar em doenças prejudiciais à saúde (LYE et al., 2013). A comercialização do leite cru em forma informal nos últimos anos vem chamando a atenção das autoridades, visto que sua produção e venda não sofrem inspeção, aumentando cada vez mais sua produção (EMBRAPA, 2012; MOLINA; CENTENARO; FURLAN, 2015).

De acordo com o Artigo 476 do RIISPOA, Brasil (1981) e a Instrução normativa nº 76, Brasil (2018), é considerado normal o leite cru refrigerado que apresenta as seguintes características centesimal (100g): teor de gordura mínima de $3 \%$, acidez titulável entre 0,14 e $0,18^{\circ} \mathrm{D}$, densidade a $15^{\circ} \mathrm{C}$ entre 1,028 e 1,034 , lactose no mínimo de 4,3\%, extrato seco total mínimo de $11,4 \%$ e extrato seco desengordurado mínimo de 8,4\% (BRASIL, 1981; BRASIL, 2018).

O leite é um ótimo meio de cultura para a propagação de micro-organismos. A preocupação com a conservação de sua qualidade deve começar mesmo antes da ordenha, com os cuidados sanitários do rebanho e os cuidados higiênicos dispensados ao local e equipamentos de ordenha. Deve ser lembrado, inclusive o papel de maior influência praticado pelas pessoas envolvidas na atividade, sem contatar na forma que o leite é repassado para os consumidos, tais como embalagens e a adequação do armazenamento. Por isso, o cuidado da qualidade higiênica do leite e uso das normas exigidas por leite. Diante disso, o objetivo desse trabalho foi avaliar os parâmetros microbiológicos e físico-químicos de leite cru in natura comercializados de forma informal nas cidades de Solânea PB e Bananeiras PB.

\section{Material e Métodos}

O experimento foi realizado no período de novembro à dezembro de 2019, no Centro de Ciências Humanas, Sociais e Agrárias, da Universidade Federal da Paraíba - UFPB, campus III, Bananeiras - PB. Nos laboratórios de Pesquisa e Desenvolvimento de Produtos Laticínios (PDLAT), no Laboratório de Microbiologia de Alimentos (LMA) e no Laboratório de Análise Físico-química de Alimentos (LAFQ).

\section{Amostragem}

A matéria-prima utilizada para a realização desse estudo foi o leite in natura comercializado informalmente em pontos de vendas localizadas em duas cidades do brejo paraibano. Foram coletadas 10 amostras de leite in natura durante 4 semanas, sendo 5 amostras na cidade de Solânea PB e 5 amostras 
na cidade de Bananeiras PB. Após a coleta, as amostras foram acondicionadas em caixas de isopor com gelo e encaminhadas ao Laboratório de Pesquisa e Desenvolvimento de Produtos Laticínios (PDLAT), prosseguindo para os demais laboratórios, para análise imediata dos parâmetros físico-químicos e microbiológicos.

\section{Avaliação Microbiológica}

As análises microbiológicas foram realizadas no Laboratório de Microbiologia de Alimentos (LMA), as amostras foram analisadas seguindo os métodos descritas no manual da American Public Health Association (APHA, 2001) e obedecendo aos padrões exigidos pela Instrução Normativa $n^{\circ} 62$ de 29 de dezembro de 2011, pelas Instruções Normativas n 76 e $\mathrm{n}^{\circ}$ 77, de 26 de dezembro de 2018, do Ministério da Agricultura Pecuária e Abastecimento - MAPA (BRASIL, 2011; BRASIL, 2018), para os parâmetros de Coliformes à $35^{\circ} \mathrm{C} \mathrm{NMP} / \mathrm{ml}$ e a $45^{\circ} \mathrm{C} \mathrm{NMP} / \mathrm{ml}$ e Bactérias Mesófilas UFC/ml.

\section{Análises Físico-Químicas}

As análises físico-químicas foram realizadas no Laboratório de Análise Físico-química de Alimentos (LAFQ), as amostras foram analisadas em triplicatas obedecendo aos padrões exigidos pela legislação da Instrução Normativa ${ }^{\circ}$ 62, de 29 de dezembro de 2011, do Ministério da Agricultura Pecuária e Abastecimento - MAPA, seguindo a metodologia do Instituto Adolfo Lutz (IAL, 2008), os parâmetros físico-químicos avaliados foram: Temperatura, teor de gordura, sólidos não gordurosos, densidade, índice do ponto crioscópico, proteína, lactose, sais minerais, acidez Dornic e pH, conforme as recomendações.

\section{Análise dos resultados}

Os dados referentes aos resultados das amostras foram analisados comparando-se as médias entre as diferentes amostras pelo Teste de médias (teste de Tukey, $\mathrm{p}<0,05$ ). As análises foram realizadas utilizando o software Statistica 7.0 (Statsoft Inc., Tulsa, OK, EUA).

\section{Resultados e Discussão}

\section{Análise Microbiológica}

Os resultados para os parâmetros microbiológicos estão representados na Tabela 1 e 2, conforme as exigências dos padrões da Instrução Normativa $n^{\circ}$ 62, de 29 de dezembro de 2011 e no 76 e $n^{\circ} 77$, de 26 de dezembro de 2018, do Ministério da Agricultura, Pecuária e Abastecimento (BRASIL, 2011; BRASIL, 2018). 
Tabela 1 - Valores médios das análises microbiológicos de amostras do município de Solânea PB

\begin{tabular}{ccccccc}
\hline $\begin{array}{c}\text { PARÂMETROS } \\
\text { MICROBIOLÓGICOS }\end{array}$ & $\mathbf{1}$ & $\mathbf{2}$ & $\mathbf{3}$ & $\mathbf{4}$ & $\mathbf{5}$ & $\begin{array}{c}\text { PADRÃO } \\
\text { (BRASIL, 2011) }\end{array}$ \\
\hline Coliformes a $35^{\circ} \mathrm{C} \mathrm{NMP/ml}$ & $4,6 \times 10^{2 \mathrm{~b}}$ & $7,5 \times 10^{2 \mathrm{a}}$ & $1,0 \times 10^{2 \mathrm{c}}$ & $4,6 \times 10^{2 \mathrm{~b}}$ & $4,6 \times 10^{2 \mathrm{~b}}$ & $1 \times 10^{3}$ \\
Coliformes a $45^{\circ} \mathrm{C} \mathrm{NMP} / \mathrm{ml}$ & $4,6 \times 10^{2 \mathrm{c}}$ & $7,4 \times 10^{3 \mathrm{ab}}$ & $3,6 \times 10^{2 \mathrm{~d}}$ & $9,3 \times 10^{2 \mathrm{a}}$ & $3,6 \times 10^{2 \mathrm{~d}}$ & $1 \times 10^{2}$ \\
Bactérias Mesófilas UFC/ml & $1,0 \times 10^{4 \mathrm{~d}}$ & $3,0 \times 10^{2 \mathrm{c}}$ & $19,9 \times 10^{2 \mathrm{a}}$ & $6,2 \times 10^{2 \mathrm{~b}}$ & $6,6 \times 10^{2 \mathrm{~b}}$ & $8 \times 10^{4}$ \\
\hline
\end{tabular}

*letras diferentes na mesma linha diferem entre si pelo teste de Tukey a nível de 5\%; NMP: Número mais provável; UFC: Unidade Formadora de Colônias;

Tabela 2 - Valores médios das análises microbiológicos de amostras do município de Bananeiras PB

\begin{tabular}{ccccccc}
\hline $\begin{array}{c}\text { PARÂMETROS } \\
\text { MICROBIOLÓGICOS }\end{array}$ & $\mathbf{6}$ & $\mathbf{7}$ & $\mathbf{8}$ & $\mathbf{9}$ & $\mathbf{1 0}$ & $\begin{array}{c}\text { PADRÃO } \\
\text { (BRASIL, 2011) }\end{array}$ \\
\hline Coliformes a $35^{\circ} \mathrm{C} \mathrm{NMP/ml}$ & $1,1 \times 10^{3 \mathrm{a}}$ & $1,1 \times 10^{3 \mathrm{a}}$ & $1,1 \times 10^{3 \mathrm{a}}$ & $1,1 \times 10^{3 \mathrm{a}}$ & $1,1 \times 10^{3 \mathrm{a}}$ & $1 \times 10^{3}$ \\
Coliformes a $45^{\circ} \mathrm{C} \mathrm{NMP} / \mathrm{ml}$ & $1,1 \times 10^{2 \mathrm{~b}}$ & $2,0 \times 10^{2 \mathrm{a}}$ & $1,0 \times 10^{2 \mathrm{bc}}$ & $1,1 \times 10^{2 \mathrm{~cd}}$ & $1,1 \times 10^{2}$ & $1 \times 10^{2}$ \\
Bactérias Mesófilas UFC/ml & $19,6 \times 103^{\mathrm{a}}$ & $14,6 \times 10^{3 \mathrm{~b}}$ & $2,0 \times 10^{3 \mathrm{c}}$ & $19,3 \times 10^{3 \mathrm{ab}}$ & $1,0 \times 10^{2}$ & $8 \times 10^{4}$
\end{tabular}

*letras diferentes na mesma linha diferem entre si pelo teste de Tukey a nível de 5\%; NMP: Número mais provável; UFC: Unidade Formadora de Colônias;

De acordo com os resultados obtidos na tabela 1 e 2 pode-se observar que as amostras da cidade de Solânea $\mathrm{PB}$, apresentaram contagens de coliformes a $35^{\circ} \mathrm{C}$ e a $45^{\circ} \mathrm{C}$ acima de $10^{3}$ e $10^{2}$, valor preconizada pela legislação da IN n ${ }^{\circ}$ 62, de 29 de dezembro de 2011 e encontrado no estudo de Farias, Croisfelt e Baffi (2014), ao comparar com o estudo de Souza, Nogueira e Nunes (2011), que obteve contaminação nas suas amostra, por possuir dados semelhantes, resultando em contaminação por coliformes em todas as amostras da tabela 1. Já para os mesmos parâmetros na tabela 2, pode observar que as amostras estavam em menor nível de contaminação, sendo tolerado $10^{3}$ e $10^{2}$.

Os resultados acima de $1 \times 10^{3}$ e $1 \times 10^{2}$ indicam controle inadequado quando a qualidade microbiológica, sendo as causas mais frequentes aquelas provenientes do leite cru, local de venda e/ou falta de manipulação com cuidados de higiene.

$\mathrm{Na}$ análise da presença de bactérias mesófilos aeróbios puderam-se observar que as amostras de número 3, 6, 7 e 9 estavam muito acima do padrão máximo exigido, ou seja, na cidade de Solânea PB apenas uma amostra estava fora do padrão, já na cidade de Bananeiras PB três amostradas estavam fora do padrão. A presença dessas bactérias é utilizada como indicador das condições sanitária do leite. Recomendando o uso e aplicação de processamento térmico e aplicação das boas práticas de fabricação.

Com base na Instrução Normativa n ${ }^{\circ} 77$, de 26 de dezembro de 2018, recomendasse que o leite seja armazenado na propriedade rural em tanques de refrigeração em temperatura máxima de $4^{\circ} \mathrm{C}$ e chegando ao local de processamento em temperatura máxima de $7^{\circ} \mathrm{C}$ (BRASIL, 2018). Durante a coleta, as amostras estavam sendo comercializadas em temperatura ambiente, essa comercialização informal em temperatura inadequada aumenta a susceptibilidade de deterioração, diminuindo a qualidade do leite com o passar do tempo. Com falta de refrigeração há uma predominância de micro-organismos mesófilos. 
O número dessas bactérias presentes no leite cru está relacionado às condições higiênicas na produção e ao tempo e temperatura em que o leite é armazenado. Sendo fundamental a contagem desse tipo de micro-organismo para identificar sua qualidade através da atividade metabólica desses microrganismos resultando em alterações bioquímicas nos constituintes do leite que diminuem a vida útil (MARIOTO et al, 2020).

O controle microbiológico do leite é de suma importância para a saúde humana, a propagação de bactérias no leite o torna impróprio para consumo, devido a alterações causada pela presença desse micro-organismo em sua composição química. Esta contaminação por microrganismos tem sido constante, trazendo problemas e perdas econômicas em produtos que não há um controle e aplicação de práticas higiênicas (PADILHA et al., 2001; ATANAGILDO, 2019).

\section{Análise Físico-química}

Os resultados para os parâmetros físico-químicos estão representados por valores médios nas Tabelas 3 e 4, conforme as exigências dos padrões da Instrução Normativa n ${ }^{\circ}$ 62, de 29 de dezembro de 2011, do Ministério da Agricultura, Pecuária e Abastecimento (BRASIL, 2011).

Tabela 3 - Valores médios das análises físico-químicas de amostras do município de Solânea PB

\begin{tabular}{cccccccc} 
AMOSTRAS & $\mathbf{1}$ & $\mathbf{2}$ & $\mathbf{3}$ & $\mathbf{4}$ & $\mathbf{5}$ & Média & Padrão Oficial* \\
\hline Temperatura $\left({ }^{\circ} \mathrm{C}\right)$ & $15,2^{\circ} \mathrm{C}$ & $24{ }^{\circ} \mathrm{C}$ & $25,5^{\circ} \mathrm{C}$ & $24,6^{\circ} \mathrm{C}$ & $18^{0} \mathrm{C}$ & $\mathbf{2 1 , \mathbf { 5 } ^ { \circ } \mathbf { C }}$ & $7-9^{\circ} \mathrm{C}$ \\
Gordura $(\%)$ & 3,55 & 3,32 & 2,49 & 3,77 & 3,75 & $\mathbf{3 , 3 8 \%}$ & Mín. $3,0 \%$ \\
Sólidos não gordurosos $(\%)$ & 8,35 & 8,81 & 8,45 & 8,06 & 8,22 & $\mathbf{8 , 3 8 \%}$ & Mín. $8,4 \%$ \\
Densidade $(\mathrm{g} / \mathrm{mL})$ & 1,031 & 1,029 & 1,029 & 1,028 & 1,027 & $\mathbf{1 , 0 2 9}$ & $1,028-1,034 \mathrm{~g} / \mathrm{ml}$ \\
Ponto crioscópico $\left({ }^{\circ} \mathrm{H}\right)$ & 0,531 & 0,534 & 0,514 & 0,502 & 0,563 & $\mathbf{0 , 5 3}{ }^{\circ} \mathbf{H}$ & $-0,530$ a $0,550^{\circ} \mathrm{H}$ \\
Proteína $(\%)$ & 3,12 & 3,11 & 2,97 & 2,98 & 3,82 & $\mathbf{3 , 2 0 \%}$ & Mín. $2,9 \%$ \\
Lactose $(\%)$ & 4,84 & 4,66 & 4,42 & 4,32 & 4,32 & $\mathbf{4 , 5 1 \%}$ & Mín. $4,3 \%$ \\
Sais minerais $(\%)$ & 0,70 & 0,70 & 0,66 & 0,66 & 0,70 & $\mathbf{0 , 6 8}$ & -- \\
Acidez $\left({ }^{\circ} \mathrm{D}\right)$ & 0,19 & 0,19 & 0,19 & 0,19 & 0,19 & $\mathbf{0 , 1 9} \mathbf{D}$ & 0,14 a $0,18{ }^{\circ} \mathrm{D}$ \\
$\mathrm{pH}$ & 7,0 & 7,11 & 6,38 & 6,71 & 6,54 & $\mathbf{6 , 7 5}$ & -- \\
\hline
\end{tabular}

Fonte: própria (2020).

De acordo com a legislação em vigor, as amostras analisadas ( Tabelas 3 e 4) quanto ao teor de gordura $(\%)$, Sólidos não gordurosos $(\%)$, Densidade $(\mathrm{g} / \mathrm{mL})$, Ponto crioscópico $\left({ }^{\circ} \mathrm{H}\right)$, Proteína $(\%)$, Lactose (\%), Sais minerais (\%), Acidez $\left({ }^{\circ} \mathrm{D}\right)$ e pH, respectivamente, não atenderam aos padrões físicoquímicos, segundo a Instrução Normativa nº 62, 76 e 77 (BRASIL, 2011; BRASIL, 2018).

Tabela 4 - Valores médios das análises físico-químicas de amostras do município de Bananeiras

\begin{tabular}{cccccccc}
\hline AMOSTRAS & $\mathbf{6}$ & $\mathbf{7}$ & $\mathbf{8}$ & $\mathbf{9}$ & $\mathbf{1 0}$ & Média & Padrão Oficial* \\
\hline Temperatura $\left({ }^{\circ} \mathrm{C}\right)$ & $15,2^{\circ} \mathrm{C}$ & $17,7^{\circ} \mathrm{C}$ & $17,9^{0} \mathrm{C}$ & $20,5^{0} \mathrm{C}$ & $18^{0} \mathrm{C}$ & $\mathbf{1 7 , 9}^{\mathbf{C}} \mathrm{C}$ & $7-9^{\circ} \mathrm{C}$ \\
Gordura $(\%)$ & 2,9 & 3,37 & 2,49 & 3,71 & 3,76 & $\mathbf{3 , 2 5 \%}$ & Mín. $3,0 \%$ \\
Sólidos não gordurosos $(\%)$ & 8,38 & 8,81 & 8,41 & 8,06 & 7,87 & $\mathbf{8 , 3 1 \%}$ & Mín. $8,4 \%$ \\
Densidade $(\mathrm{g} / \mathrm{mL})$ & 1,032 & 1,029 & 1,029 & 1,028 & 1,028 & $\mathbf{1 , 0 2 9}$ & $1,028-1,034 \mathrm{~g} / \mathrm{ml}$
\end{tabular}




\begin{tabular}{|c|c|c|c|c|c|c|c|}
\hline Ponto crioscópico $\left({ }^{\circ} \mathrm{H}\right)$ & $-0,557$ & $-0,533$ & $-0,504$ & $-0,502$ & $-0,491$ & $-0,517^{\circ} \mathrm{H}$ & $-0,530$ a $-0,550^{\circ} \mathrm{H}$ \\
\hline Proteína (\%) & 3,25 & 3,10 & 2,97 & 2,98 & 2,89 & $3,04 \%$ & Mín. 2,9\% \\
\hline Lactose $(\%)$ & 4,82 & 4,60 & 4,42 & 4,34 & 4,27 & $4,49 \%$ & Mín. 4,3\% \\
\hline Sais minerais $(\%)$ & 0,73 & 0,70 & 0,66 & 0,66 & 0,66 & $0,68 \%$ & --- \\
\hline Acidez $\left({ }^{\circ} \mathrm{D}\right)$ & 0,18 & 19 & 19 & 19 & 19 & $0,19^{\circ} \mathrm{D}$ & 0,14 a $0,18^{\circ} \mathrm{D}$ \\
\hline $\mathrm{pH}$ & 6,39 & 6,25 & 6,33 & 6,29 & 6,48 & 6,35 & --- \\
\hline
\end{tabular}

Fonte: própria (2020).

Para as análises físico-químicas do leite coletado nos municípios de Solânea PB e Bananeiras $\mathrm{PB}$, foram obtidos os seguintes resultados: a temperatura encontrada foi entre $15^{\circ} \mathrm{C}$ a $25^{\circ} \mathrm{C}$ entre todas as amostras, com médias de $21,5^{\circ} \mathrm{C}$ para as 5 amostras da cidade de Solânea $\mathrm{PB}$ e $17,9^{\circ} \mathrm{C}$ para as 5 amostras na cidade de Bananeiras PB. Em quer a amostra número 3 coletada em Solânea diferiu significativamente a nível de 5\% das demais, ou seja, foi a maior temperatura encontrada dentre as amostras. A Instrução Normativa n $^{\circ}$ 77, de 26 de dezembro de 2018, Brasil (2018), recomenda que o leite chegue ao laticínio, ou local de processamento, à temperatura de no limite mínimo de $7,0^{\circ} \mathrm{C}$ e no máximo $9,0^{\circ} \mathrm{C}$. Ao relacionar essa informação com os dados coletados todas as amostras, no momento da coleta, estavam sendo comercializadas sem refrigeração adequada ou nenhuma, consequentemente, as temperaturas encontradas aproximam-se da temperatura ambiente, ou seja, todas as amostras estavam fora do padrão exigido. Ao comparar com estudo de Atanagildo (2019), os resultados variaram de 2,8 (mínima) a $22,8^{\circ} \mathrm{C}$ (máxima), com média de temperatura de $9,53^{\circ} \mathrm{C}$ em 7 amostras, com resultado de média abaixo do encontrado nesse estudo.

Para a análise de gordura os resultados variaram em médias de $3,38 \%$ a 3,25\%, o que segundo a Instrução Normativa nº 76, de 26 de dezembro de 2018, Brasil (2018), o teor de gordura varia de acordo com a classificação do leite em integral, semidesnatado e desnatado, sendo respectivamente os padrões preconizados de no mínimo 3,0\% de gordura em leite cru refrigerado (BRASIL, 2011; BRASIL, 2018). Porém o teor de gordura analisado nas amostras 3, 6 e 8 estão em desacordo com a legislação, esses resultados demonstraram que o valor encontrado de gordura pode ser relacionado à padronização do leite, e o baixo teor de gordura pode determinar a fraude com adição de água, que consequentemente, resulta na diminuição do extrato seco desengordurado. Vale ressaltar, que para os produtores de leite que não são fiscalizados pelos órgãos competentes, não há controle da padronização do teor de gordura do leite para 3,0\%.

Para análise de teor de sólidos não gordurosos ou extrato seco desengordurado, os resultados foram de valores médios de 8,38\% e 8,31\%, apenas as amostras 2,3,7 e 8, estão dentro do padrão exigido, que é no mínimo 8,4\%. Porém ao comparar com o resultado encontrado por Amaral e Santos (2011), com média de 7,5\% em 3 amostras, que estavam em total desacordo com a legislação, os resultados desse estudo estavam acima da média encontrada em ambos locais.

Para a análise de densidade relativa, conforme o art. $5^{\circ}$ da Instrução Normativa $n^{\circ} 76$, de 26 de novembro de 2018, do Ministério da Agricultura, Pecuária e Abastecimento, os resultados encontrados foram de médias iguais para ambos os locais, de $1,029 \mathrm{~g} / \mathrm{ml}$. Portanto, a média está dentro do valor 
permitido pela legislação. A densidade do leite deve apresentar-se entre $1,028 \mathrm{~g} / \mathrm{ml}$ e $1,034 \mathrm{~g} / \mathrm{ml}$ à $15^{\circ} \mathrm{C}$, segundo recomendação da legislação vigente (BRASIL, 2018). Valores abaixo dessa faixa podem indicar adulteração, por adição de água, e para valores acima do preconizado, adulteração por adição de outras substâncias ou desnate do leite (POLEGATO; RUDGE, 2003; ALVES; ANTES, 2014).

Para análise de Ponto crioscópico ou índice crioscópico que é a medida do ponto de congelamento do leite ou da depressão do ponto de congelamento do leite em relação ao da água, os resultados variaram em médias de $-0,517$ e $-0,530^{\circ} \mathrm{H}$, sendo aceitável pela legislação o ponto crioscópico do leite entre $0,530^{\circ} \mathrm{H} \mathrm{e}-0,550^{\circ} \mathrm{H}$ (BRASIL, 2018). Havendo diferença significativa a nível de $5 \%$ entre as amostras 5, 2 e 1 das demais, em Solânea PB e diferença entre as amostras 6 e 7 em Bananeiras PB, em quer ambas estavam dentro do valor mínimo exigido. Segundo Castanheira (2010), resultados acima de $0,530^{\circ} \mathrm{H}$ significam acréscimo de água e resultados abaixo de $-0,550{ }^{\circ} \mathrm{H}$, indicam possíveis problemas pelo congelamento do leite no tanque de expansão, além de adulteração do leite por reconstituintes. Esse tipo de adulteração em amostras de leite comercializadas informalmente foi evidenciado no estudo de Polegato e Rudge (2003), Silveira e Bertagnolli (2014), também encontraram valores inadequados para o ponto crioscópico de amostras de leite cru analisadas. Com esses resultados se mostra claramente o descuido e imprudência por parte de muitos produtores de leite que fornecem leite nesse tipo de comercialização pela região, pois além de não ter um fornecedor que garanta a qualidade da matériaprima, a fraude é evidente e não existe um controle de qualidade (BRASIL, 2018).

Para a análise de teor de proteína os resultados variaram com médias de 3,20\% em Solânea PB e 3,04\% em Bananeiras PB, resultando em todas as amostras em confronto com a legislação estando dentro do padrão mínimo exigido, que é 2,9\% de proteína para leite cru refrigerado (BRASIL, 2018). No estudo de Bylund (2003), o teor variou entre 3,0\% e 3,8\%, sendo semelhante aos valores médios encontrados nesse estudo.

Para análise de lactose os resultados variaram em médias de 4,51\% e 4,49\%. De acordo com o Artigo 476 do RIISPOA (BRASIL, 1997), considera-se normal o leite que apresenta no mínimo 4,3\% de lactose. Sendo assim, o teor de lactose das amostras de leite analisadas pode ser considerado normal, porém, apenas o teor da amostra de número 10 estava abaixo do valor mínimo exigido, com teor de $4,27 \%$.

Para análise de sais minerais os resultados variaram em médias de $0,68 \%$ a $0,71 \%$. Não é estabelecido teor de sais minerais em leite, mas é possível observar que não houve grande variação entre as amostras. Amaral e Santos (2011) encontraram em amostras de leite cru comercializado na cidade de

Solânea-PB, valores para minerais variando entre 0,57 e 0,64 , ou seja, foram encontrados valores abaixo do desse estudo.

Para análise de acidez ${ }^{\circ}$ Dornic, os resultados da maioria das amostras permaneceram em $19^{\circ} \mathrm{D}$ $(0,16 \mathrm{~g}$ de ácido lático/100g de produto) para acidez Dornic, onde apenas a amostra 6 , da cidade de Bananeiras PB, está dentro dos padrões, que estabelece o limite máximo de $18^{\circ} \mathrm{D}$, sendo que foram encontrados valores de até $19^{\circ} \mathrm{D}$ em outras amostras. Esses resultados indicam que provavelmente não 
houve uma refrigeração imediata logo após a ordenha, ou ainda, devido à falta de higiene durante a obtenção higiênica, conforme é exigido pela Instrução Normativa $\mathrm{n}^{\circ} 77$, e a alta acidez pode ocorrer da acidificação da lactose, proveniente da multiplicação de micro-organismos deterioradores ou patogênicos (BRASIL, 2018).

No estudo de Silveira e Bertagnolli (2014) identificaram que em torno de $80 \%$ das amostras analisadas de leite cru comercializadas informalmente em Santa Maria - RS, apresentaram acidez superior a $0,18 \mathrm{~g}$ de ácido lático/100 $\mathrm{mL}$. O alto grau de acidez no leite é o resultado da fermentação da lactose provocada pela ação de enzimas e multiplicação de bactérias lácticas, assim também com ocasionar a coagulação da caseína e assim, limitando o seu uso (BJORKROTH; KOORT, 2011). Segundo Mareze et. al (2015) e Atanagildo (2019), o bicarbonato de sódio, é o componente mais utilizado como redutor de acidez em leite, porém conforme a legislação Brasil (2018), não é permitida a adição de conservantes, substâncias capazes de corrigir o pH ou aditivos no leite cru.

Para análise de $\mathrm{pH}$ os resultados variaram em médias de 6,75 e 6,35, não possuindo tanta variação nas amostras da cidade de Bananeiras PB, diferente do da cidade de Solânea PB, em quer as amostras 1 e 2 diferiram significativamente entre as demais a nível de 5\% A água apresenta $\mathrm{pH}$ próximo da neutralidade e quando adicionada ao leite aumenta significativamente o seu pH. Entretanto a legislação não preconiza valores de $\mathrm{pH}$, sendo que valores com muito diferencial entre si resultam da fraude com água ou há relação entre o pH do leite com a saúde da glândula mamária, onde animais com a mastite, apresentam um leite com pH levemente alcalino $(7,3$ a 7,5), podendo então ser usado como parâmetro indicativo de sanidade, pois o $\mathrm{pH}$ do leite cru varia entre 6,6-6,8 a temperatura de $20^{\circ} \mathrm{C}$ (RODAS et al., 2014; ATANAGILDO, 2019).

Importante frisar que essas amostras são destinadas ao comercio informal de Solânea e Bananeiras e, entre tanto, os seus fornecedores ou produtores devem produzir um alimento seguro e com qualidade, não somente pelo valor nutricional, como também no aspecto de higiene. Estes fatores são de grande importância, pois o público consumidor deste alimento é bastante susceptível a enfermidades transmitidas por alimentos.

As pequenas variações nos componentes do leite podem ser explicadas pela ordem genética, fisiológica, ambiental e ainda pode ser devido ao desnate e fraudes para aumentar o rendimento (MENDES et al., 2010). De acordo com as análises físico-químicas e microbiológicas realizadas, constatou-se a necessidade de refrigeração e cuidados com a obtenção e transporte do leite informal, que resulta em acidificação do produto e contagens acima do permitido pela legislação vigente. Considerando a comercialização informal e inadequada do leite, os resultados evidenciam a precariedade do produto, pois do total de amostras analisadas, todas apresentaram-se fora dos padrões quanto às análises microbiológicas e físico-químicas, respectivamente, entre as características físicoquímicas avaliadas. 


\section{Conclusões}

De acordo com os resultados obtidos neste trabalho, concluiu-se que o leite cru comercializado de forma informalizada nas cidades de Solânea PB e Bananeiras PB no período desse estudo onde todas as amostras apresentaram-se fora dos padrões em relação à pelo menos um parâmetro de análises microbiológicas e físico-químicas, respectivamente permitido por legislação, ou seja, não atende a todos os requisitos microbiológicos e físico-químicos, apresentando diferenças quanto sua composição físicoquímica e qualidade microbiológica.

Vale ressaltar que mediante os resultando encontrados o leite comercializado nesses locais está fora do padrão de consumo, além de apresentarem características para adulteração da composição, podendo causar riscos à saúde do consumidor, evidenciando o perigo de consumo do leite informal. Sendo necessário a aplicação de fiscalização pelos órgãos competentes afim de inibir a venda desse e envolver instituições capazes de conscientizar esses tipos de produtores e fornecedores, com aplicação de ferramentas para capacitação dos mesmos e com isso melhorar o desempenho e a obtenção de leite de qualidade pelo produtor junto ao consumidor e aos órgãos de fiscalização.

\section{Referências}

FARIAS, C. P.; CROISFELT, F. M.; BAFFI, M. A. Microbiological quality of in natura raw milk, raw chilled and pasteurized milk sold in the region of Uberlândia, MG. Revista Verde de Agroecologia e Desenvolvimento Sustentável, Pombal - Pb, v. 9, n. 4, p. 250-254, out-dez, 2014.

AMARAL, C. R. S.; SANTOS, E. P. Leite cru comercializada na cidade de Solânea, PB: caracterização físico-química e microbiológica. Revista Brasileira de Produtos Agroindustriais, 13 (1), 7-13. 2011.https://doi.org/10.15871/1517-8595/rbpa.v13n1p7-13.

APHA, American Public Health Association. Compendium of Methods for the Microbiological Examination of foods. 3a ed. Washington: APHA; 1992.

BJÖRKROTH, J.; KOORT, J. Taxonomy and biodiversity. In: Encyclopedia of Dairy Sciences. Elsevier Scientific Publ. Co, 2011. p. 45-48.

BRASIL, Ministério da Agricultura, Pecuária e Abastecimento - MAPA Instrução Normativa $n^{\circ} 51$, de 18 de setembro de 2002. Aprova os Regulamentos Técnicos de Produção, Identidade e Qualidade do Leite tipo A, do Leite tipo B, do Leite tipo C, do Leite Pasteurizado e do Leite Cru Refrigerado e o Regulamento Técnico da Coleta de Leite Cru Refrigerado e seu Transporte a Granel. Diário Oficial da União, Brasília, p.13, 21 set. 2002. Seção 1.

BRASIL, Ministério da Agricultura, Pecuária e Abastecimento - MAPA. Instrução Normativa nº 77, de 26 de novembro de 2018. Critérios e procedimentos para a produção, acondicionamento, conservação, 
transporte, seleção e recepção do leite cru em estabelecimentos registrados no serviço de inspeção oficial. Diário Oficial da União, Brasília, p. 10, 26 nov. 2018. Edição 230, Seção 1.

BRASIL, Ministério da Agricultura, Pecuária e Abastecimento - MAPA. Instrução Normativa nº 76, de 26 de novembro de 2018. Regulamentos Técnicos que fixam a identidade e as características de qualidade que devem apresentar o leite cru refrigerado, o leite pasteurizado e o leite pasteurizado tipo A. Diário Oficial da União, Brasília, p. 9, 30 nov. 2018. Edição: 230. Seção: 1.

BRASIL, Ministério da Agricultura, Pecuária e Abastecimento. Instrução - MAPA. Normativa nº 62, de 29 de dezembro de 2011. Aprova o Regulamento Técnico de Produção, Identidade e Qualidade do Leite tipo A, o Regulamento Técnico de Identidade e Qualidade de Leite Cru Refrigerado, o Regulamento Técnico de Identidade e Qualidade de Leite Pasteurizado e o Regulamento Técnico da Coleta de Leite Cru Refrigerado e seu Transporte a Granel, em conformidade com os Anexos desta Instrução Normativa. Diário Oficial da União, Brasília, 31 dez. de 2011. Seção 1, p. 6.

BRASIL, Ministério da Agricultura. Secretaria Nacional de Defesa Agropecuária. Laboratório Nacional de Referência Animal. Regulamento Industrial de Inspeção Sanitária de Produtos de Origem Animal: artigo 476. Brasília: Ministério da Agricultura, 1981.

BYLUND, G. Dairy processing handbook. Tetra Pak Processing Systems AB, 2003.

DIAS, J. A.; ANTES, F. G. Qualidade físico-química, higiênico-sanitária e composicional do leite cru: indicadores e aplicações práticas da Instrução Normativa 62. Documentos 158. Porto Velho, RO: Embrapa Rondônia, ISSN 0103-9865, out., 2014.

EMBRAPA, Empresa Brasileira de Pesquisa Agropecuária Gado de Leite. Produção, industrialização e comercialização. Juiz de Fora: Embrapa Gado de Leite. 2012.

FERREIRA, A. G. G.; LYRA, D. G.; SILVA, J. C. S.; SOARES, F. M. F.; ARAÚJO, C. A. Perfil dos consumidores de leite bovino in natura no município de Santana do Ipanema - Alagoas. Nutri-time Revista Eletrônica, on-line, Viçosa, v.14, n.4, p.6056-6065, jul./ago, 2017. ISSN: 1983-9006.

IAL, Instituto Adolfo Lutz. Métodos físico-químicos para análise de alimentos. São Paulo: Instituto Adolfo Lutz, 2008. 1020p.

LYE, Y. L.; AFSAH-HEJRI, L.; CHANG, W. S.; LOO, Y. Y.; PUSPANADAN, S.; KUAN, C. H.; GOH, S. G.; SHAHRIL, N.; RUKAYADI, Y.; KHATIB, A.; JOHN, Y.H.T.; NISHIBUCHI, M.; NAKAGUCHI, Y.; SON, R. Risk of Escherichia coli O157:H7 transmission linked to the consuption of raw milk. International Food Research Journal, Serdang, v. 20, n. 2, p. 1001-1005, 2013. 
MAREZE, J.; MARIOTO, L.R.M.; GONZAGA, N.; DANIEL, G. C.; TAMANINI, R.; BELOTI, V. Detecção de adulterações do leite pasteurizado por meio de provas oficiais. Semina: Ciênc. Biol. Saúde, v. 36, n. 1 supl, 2015.

MENDES, C. G.; SAKAMOTO, S. M.; SILVA, J. B. A.; JÁCOME, C. G. M.; LEITE, A. I. Análises físico-químicas e pesquisa de fraude no leite informal comercializado no município de Mossoró, RN. Revista Ciência Animal Brasileira, Goiânia, v. 11, n. 2, p. 349-356, abr./jun. 2010.

MOLINA, C. H. A.; CENTENARO, G. S.; FURLAN, V. J. M. Qualidade do leite cru comercializado informalmente no município de Itaqui-RS. Vigilância Sanitária debate. 2015;3(4):106-113.

MONTANHINI, T. M. M.; HEIN, K. Qualidade do leite cru comercializado informalmente no município de Piraí do Sul, estado do Paraná, Brasil. Revista Instituto Laticínios Cândido Tostes, Juiz de Fora, v. 68, n. 393, p. 10-14, jul/ago., 2013.

MOTTA, R.G.; SILVA, A.V.; GIUFFRIDA, R.; SIQUEIRA, A.K.; PAES, A.C.; MOTTA, I.G.; LISTONI; F.J.P.; RIBEIRO M.G. Indicadores de qualidade e composição de leite informal comercializado na região Sudeste do Estado de São Paulo. Pesquisa Veterinária Brasileira. 35(5):417423. 2015.

ORDÓÑEZ, Juan A. et al. Tecnologia de Alimentos: Alimentos de Origem Animal. Porto Alegre: Artmed, 2005. v. 2.

POLEGATO; E. P. S.; RUDGE; A. C. Estudo das características físico-químicas e microbiológicas dos leites produzidos por mini-usinas da região de Marília - São Paulo. Revista Higiene Alimentar, v. 17, n. 110, p. 56-63, 2003. Disponível em: <http://www.bases. bireme.br/cgi-bin/wxislind.exe/iah/ online/?.>. Acesso em: 07 mar. 2020.

RODAS, M.A.B.; SATO, J.H.S.; TAKARHASHI, A.A.; TEMERLOGLOU, D.P.; SEPAROVIC, L.; NARDINI, G. S. Leite Pasteurizado e ultra alta temperatura (UAT): Avaliação do índice crisoscópio e valor de Ph- Bol. Inst Adolfo Lutz. P. 57-59, 2014

SILVEIRA, M. L. R.; BERTAGNOLLI, S. M. M. Avaliação da qualidade do leite cru comercializado informalmente em feiras livres no município de Santa Maria-RS. Revista Vigilância Sanitária em Debate: Sociedade, Ciência \& Tecnologia (Visa em Debate), vol.2, n.2, p.75-80. 2014.

SOUZA, F.M.; NOGUEIRA, M. S.; NUNES, F. C. Qualidade microbiológica do leite cru comercializado informalmente na cidade de Areia-PB. Revista Agropecuária Técnica. Areia PB, v. 32, n. 1, p $168-171,2011$ 
TANAGILDO, L. F. Qualidade do leite comercializado em feiras livres no município de VilhenaRO. 2019. 25 f. TCC (Graduação) - Curso de Zootecnia, Faculdade da Amazônia, Vilhena RO, 2019.

TRINDADE, L. C. A.; MARTINS, M. L.; MARTINS, J. M.; MARTINS, A. D. O. Qualidade de leite cru comercializado informalmente no município de RIO POMBA, MG. Revista Higiene Alimentar, vol.32, nº 284/285, set-out. p. 72-76. 2018.

VIDAL, A. M. C.; NETTO, A. S. Obtenção e processamento do leite e derivados. Faculdade de Zootecnia e Engenharia de Alimentos da Universidade de São Paulo, USP, Pirassununga-SP, 2018. 220 p.

VIEIRA, M.C.M.; ANDRÉ M.C.D.P.B.; SERAFINE, A.B.; LIMA, S.V.; SILVA, E.V. Avaliação microbiológica do leite pasteurizado tipo $\mathrm{C}$ comercializado no estado de Goiás no período de janeiro a junho de 2000. Revista Higiene Alimentar, n.82. p.72, 2001. 